\title{
Thermally-stimulated spontaneous currents from metal-iodine doped polyvinyl pyrrolidone-metal system
}

\author{
P K KHARE*, SANDEEP K JAIN ${ }^{\dagger}$ and SUNIL K PALIWAL \\ Department of Postgraduate Studies and Research in Physics, Rani Durgavati Vishwavidyalaya, \\ Jabalpur 482001, India \\ 'Central Instrumentation Lab, Dr H S Gaur University, Sagar 470003, India \\ MS received 21 January 1997; revised 21 July 1997
}

\begin{abstract}
A study of the spontaneous response currents from the metal-iodine doped polyvinyl pyrrolidone-metal (MPM) systems, on thermal stimulation at a constant rate, has been made with similar ( $\mathrm{Al}-\mathrm{Al}, \mathrm{Ag}-\mathrm{Ag}$ and $\mathrm{Au}-\mathrm{Au})$ and dissimilar $(\mathrm{Al}-\mathrm{Cu} / \mathrm{Ag} / \mathrm{Ni} / \mathrm{PbZn})$ electrode systems. Thermograms of spontaneous current emission of iodine-doped PVP films exhibit two maxima around $90 \pm 10^{\circ} \mathrm{C}$ and $130-160^{\circ} \mathrm{C}$ in the first heating run, whereas with the second heating run a single peak is found around $140-170^{\circ} \mathrm{C}$. The magnitude and direction of current depend on the choice and combination of electrode materials. The position of the current peak in the thermal spectrum shifts with different heating run. A temperature dependence of open-circuit voltage (OCV) is also reported and it was found that OCV varied linearly with the difference in electrode work functions. The active centres of PVP are the carbonyl group of double-bond tertiary nitrogen atom $(>\mathrm{N}-\mathrm{C}=\mathrm{O})$, and thus the charge transfer complexes are formed with iodine in PVP. The spontaneously-generated current is discussed in terms of weak complex formation with the water molecules and the liberation of different types of charges.
\end{abstract}

Keywords. Spontaneous current emission; polyvinyl pyrrolidone; active centres; charge transfer complex.

\section{Introduction}

With rapid technological advances in the preparation of films with controlled, reproducible and well defined structure, thin films are expected to play an increasingly important role in the studies of variety of solid state phenomena of basic and practical interest. It has been reported (Khare and Srivastava 1992a) that the phenomenon of spontaneous current emission (SCE) is most prominent in unannealed hygroscopic substances like polyvinyl pyrrolidone (PVP). The nature and magnitude of SCE depend upon the combinations of electrode materials. Polymers whose glass transition temperature are in higher temperature ranges possess several mechanical and dielectric relaxations at certain fixed temperature range. PVP is increasingly becoming important in pharmaceutical sciences because it can form complexes with various drugs (Khare et al 1993). Therefore, it is necessary to undertake the spontaneous current emission (SCE) phenomena of PVP, specially in the form of thin films (Khare et al 1992a). The complexes of iodine with PVP are being marketed because of their excellent germicidal properties combining the greatly reduced toxicity and absence of staining action of iodine. In this paper the experimental results of SCE of iodine-doped PVP with different combinations of vacuum-coated electrodes are discussed to elucidate the mechanism of origin and nature of SCE, which may be interesting from scientific and technological points of view.

*For correspondence 


\section{Experimental}

Commercial grade PVP was supplied by Glaxo Chemicals, Bombay with an average molecular weight 40,000 . Isothermal immersion technique was utilized for preparing thin films of iodine-doped PVP. The solution of a particular concentration was prepared in a glass beaker by first dissolving PVP $(4 \mathrm{~g})$, in $50 \mathrm{ml}$ of chloroform at room temperature. An appropriate amount of iodine was then mixed with it. The solution was continuously stirred for $30 \mathrm{~min}$ by means of a teflon-coated magnetic stirrer. Thereafter, it was stirred and heated to $60^{\circ} \mathrm{C}$ to yield a homogeneous solution. The glass beaker containing the solution was then immersed in a constant temperature oil bath. Ultrasonically-cleaned, vacuum-metallized microscopic glass slides were immersed vertically into the solution for a period of about $30 \mathrm{~min}$. After deposition of the film, glass slide was taken out and dried in an oven at $40^{\circ} \mathrm{C}$ for $24 \mathrm{~h}$. This was followed by room temperature outgassing at $10^{-5}$ torr for a further period of $24 \mathrm{~h}$. The concentration of iodine was varied from $0.5-2 \cdot 0 \mathrm{wt} \%$ to obtain films of different compositions with aluminium electrodes only. The upper metal electrode was also vacuum deposited. Aluminium, copper, silver, zinc, lead, nickel and gold metals were vacuum-deposited on the surface of the film to obtain a sandwich configuration. The following electrode combinations were used: $\mathrm{Al}-\mathrm{Al}, \mathrm{Ag}-\mathrm{Ag}, \mathrm{Au}-\mathrm{Au}, \mathrm{Al}-\mathrm{Zn}$, $\mathrm{Al}-\mathrm{Pb}, \mathrm{Al}-\mathrm{Ni}$ and $\mathrm{Al}-\mathrm{Cu}$. The thickness of the samples was $20 \mu \mathrm{m}$, which was estimated by

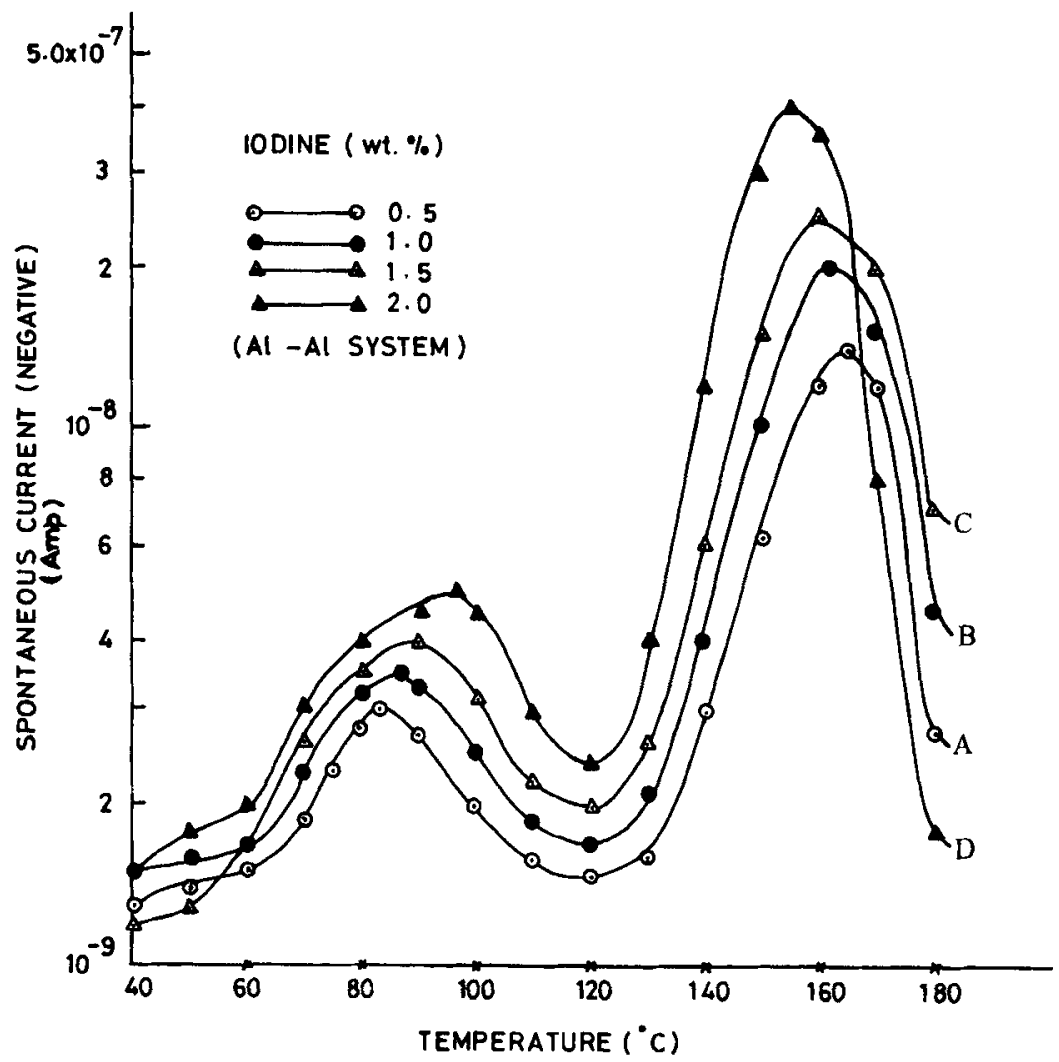

Figure 1. Effect of iodine concentration on SCE spectra of iodine-doped polyvinyl pyrrolidone films (Al-Al system). 


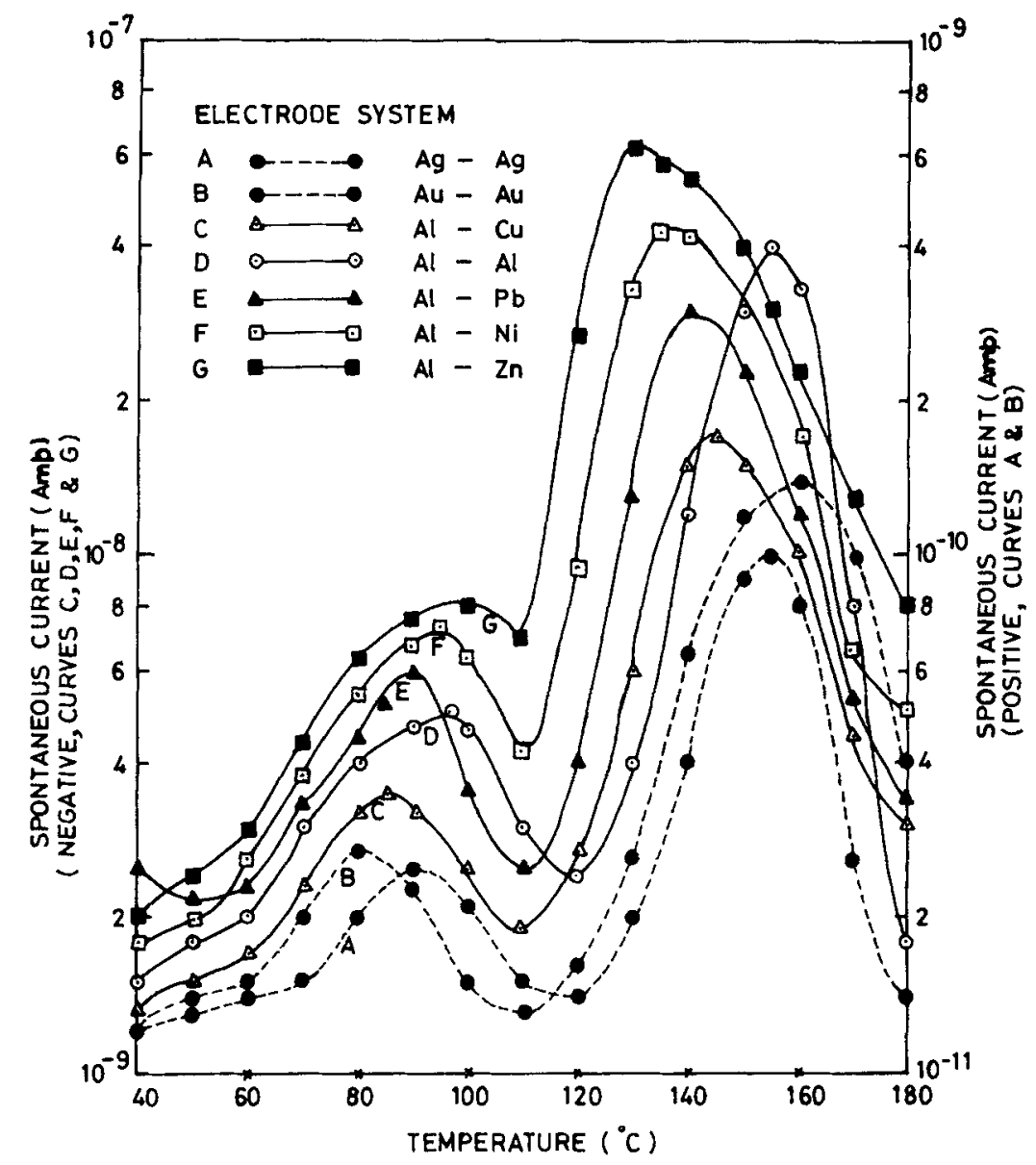

Figure 2. Spontaneous current during first heating run for metal-iodine doped polyvinyl pyrrolidone-metal systems.

measuring the capacitance of the fabricated sandwiches taking the value of dielectric constant $\varepsilon$ of PVP as 3. The output from samples was fed to an electrometer amplifier $(600 \mathrm{~B})$ for measuring current and open circuit voltages (which was carefully shielded and grounded to avoid ground loops or extraneous electrical noise) at a linear heating rate of $4 \mathrm{~K} / \mathrm{min}$ throughout the heating span.

\section{Results and discussion}

PVP samples doped with iodine are heated in the range $40-180^{\circ} \mathrm{C}$, with a uniform heating rate of $4 \mathrm{~K} / \mathrm{min}$. Figure 1 depicts the SCE thermograms for iodine-doped PVP samples of different compositions; curves $\mathrm{A}-\mathrm{D}$ correspond to the spectra of sensitized samples doped with different iodine concentrations $(0.5,1.0,1.5$ and $2.0 \mathrm{wt} \%)$. Two distinct peaks are observed around $90 \pm 7^{\circ} \mathrm{C}$ and $160 \pm 5^{\circ} \mathrm{C}$. As concentration of iodine increases, an increase in peak current is observed with a regular shifting of both the peaks.

In figure 2, SCE thermogram is shown for $2.0 \mathrm{wt} \%$ iodine-doped PVP film with $\mathrm{Au}-\mathrm{Au}$, $\mathrm{Ag}-\mathrm{Ag}, \mathrm{Al}-\mathrm{Al}, \mathrm{Al}-\mathrm{Zn}, \mathrm{Al}-\mathrm{Pb}, \mathrm{Al}-\mathrm{Ni}$ and $\mathrm{Al}-\mathrm{Cu}$ structure. In SCE of these samples the 
two well-resolved peaks have been observed in distinct temperature ranges $80-100^{\circ} \mathrm{C}$ and $130-160^{\circ} \mathrm{C}$. The order of current for peak $\mathrm{I}$ is found in between $10^{-11}-10^{-10} \mathrm{~A}$. The peak II has been found with a higher order of current which varies from $10^{-9}-10^{-7} \mathrm{~A}$. The SCE for $\mathrm{Al} / \mathrm{Zn} / \mathrm{Ag} / \mathrm{Pb} / \mathrm{Ni} / \mathrm{Cu}$ has been observed in negative direction (i.e. current flows from lower electrode to the upper electrode through the external circuit) and in the case of $\mathrm{Ag}-\mathrm{Ag}$ and $\mathrm{Au}-\mathrm{Au}$, the direction of current is found to be positive (i.e. it flows through upper electrode to the lower electrode from external circuit). Both have been shown in the same figure. The $\mathrm{Y}$-axis representing the current in positive direction has been shown on right hand side marked as $\mathrm{A}$ and $\mathrm{B}$ on the graph, while that for negative direction has been shown on left hand side marked as C, D, E, F and G.

The spontaneous currents have been recorded with four successive heating runs. However, in the present paper results only with two (first and second) heating runs have been presented.

The different curves in figure 3 exhibit the SCE for second heating run. The current found in second heating run gets reduced with all types of electrode systems. First peak in the SCE thermogram is absent and position of second peak is shifted towards higher

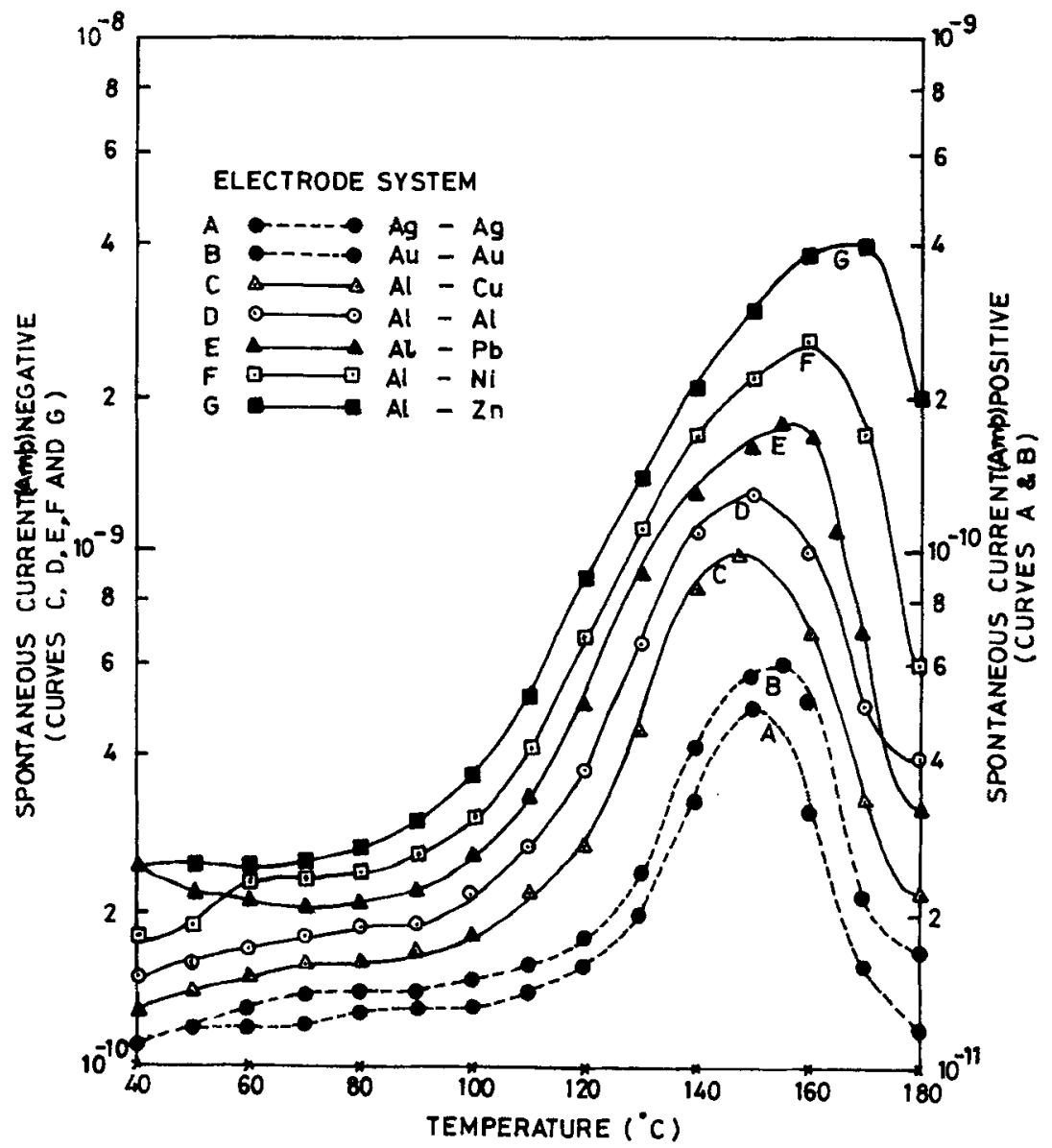

Figure 3. Spontaneous current during second heating run for metal-iodine doped polyvinyl pyrrolidone-metal systems. 


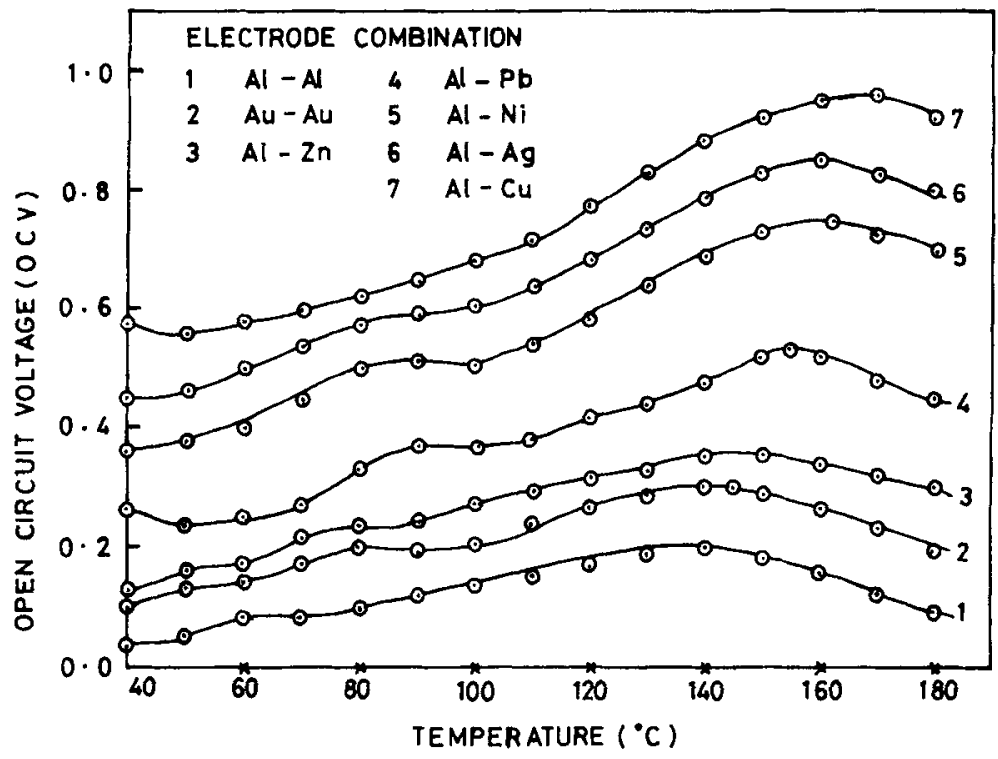

Figure 4. Open circuit voltage (OCV) during first heating for different electrode combinations.

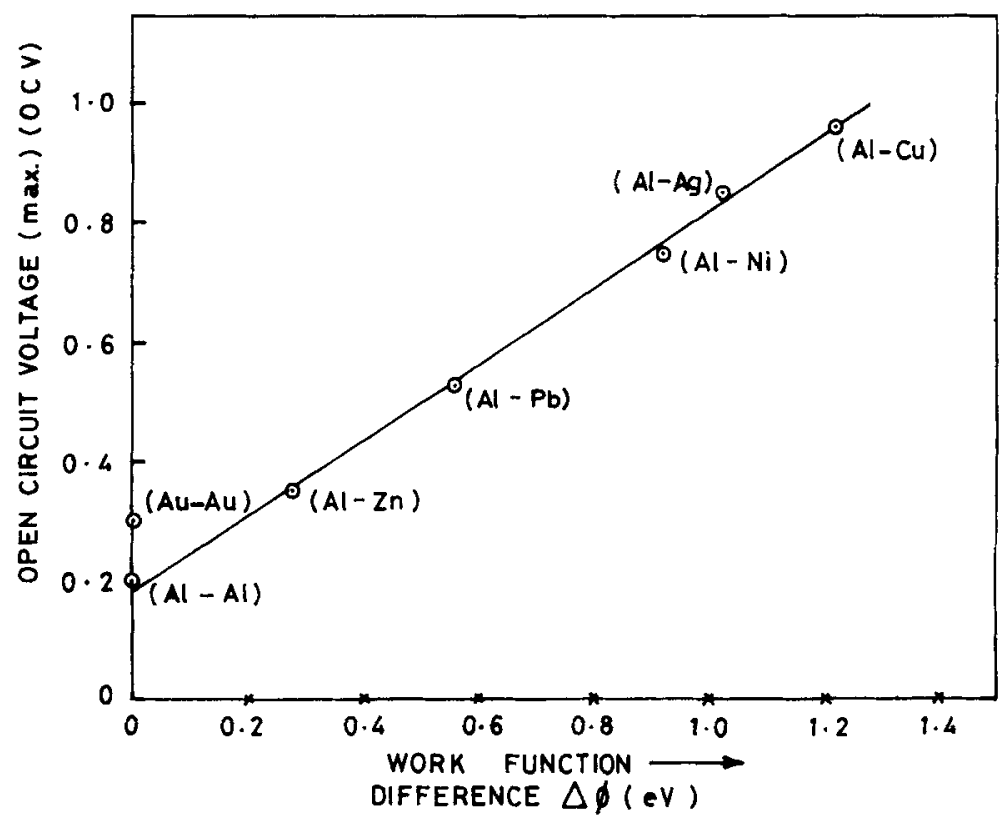

Figure 5. The peak value of $\mathrm{OCV}$ for each of the curves of figure 4 vs the work function difference of the corresponding electrodes.

temperature side. A similar behaviour is observed when the specimen is subjected to the third and fourth runs. After the second heating the current and peak positions are not changed to any significant extent.

Temperature dependence of $\mathrm{OCV}$ during the first heating run of $\mathrm{Al}-\mathrm{Zn} / \mathrm{Pb} / \mathrm{Ag} / \mathrm{Ni} / \mathrm{Cu}$ systems are shown in figure 4 . Open circuit voltage first increases with temperature, 
exhibiting a broad peak around $140-170^{\circ} \mathrm{C}$, then it tends to become steady. The peak $\mathrm{OCV}$ for each of the curves of figure 4 is plotted against the work function difference of the corresponding electrodes. It is clear from the figure that OCV magnitudes as well as sharpness of transitional peaks are proportional to the work function difference of the electrode metals.

PVP is a polar polymer, due to its carbonyl group of double-bond in the pyrrolidone ring (Tan and Challa 1976). The network structure of PVP has different lengths of $\pi$ and $\sigma$ bonds in between nitrogen, oxygen, carbon and hydrogen atoms (Gyoory 1965; Bandrup and Immergut 1975). There are several examples of apparent adsorption of PVP on a variety of substances (Elay and Spirey 1962; Chatterjee and Sethi 1983). In the case of iodine doping in PVP samples the adsorption becomes sufficiently strong to form a complex. In the present case, peaks I and II in SCE measurements are attributed to primary and secondary relaxation processes (Khare and Srivastava 1992b) alongwith the effects of complex forming conditions. The exact nature of charge transfer complexes (CTC) between PVP and iodine can be explained in terms of donating nature of conjugate pair of $\pi$ electrons to the acceptor iodine atoms. A regular pattern of shifting of peak current with increasing iodine concentrations may also suggest the strong possibility of CTC between them as follows

$$
D A=D^{+}+A^{-} \text {. }
$$

The possibilities of CTC formation between PVP and iodine may be explained as follows (i) by accepting the electrons by carbonyl group of double bond, and (ii) iodine may also get absorbed strongly with the nitrogen atom of PVP due to reactiveness of amide bond. The groups are interlinked with the amide and carbonyl bonds, hence the iodine may undergo strong CTC formation after the side group rotation. The possibility of latter mechanism is limited to certain higher temperature range where the structure is loosened to some extent and this may be observed as a peak signal in SCE measurements.

In PVP, the different phases of amorphous and crystalline regions are entangled into each other (Khare and Jain 1997). These different phases have different energy levels and by doping these boundary phases may get interconnected with each other by reducing the difference in energy levels. The motion of charge carriers is, therefore, impeded in these crystalline-amorphous interfaces and the dopant iodine may exist in complex formation as $I_{2}$ or as molecular aggregates (Khare et al 1993, 1994a).

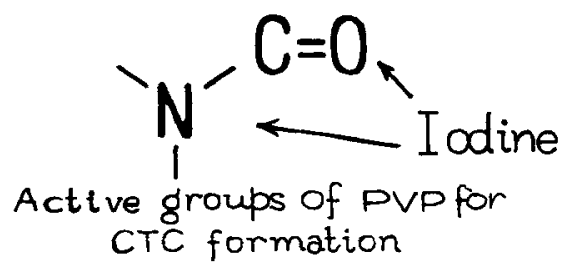

The spontaneous current measurement involves only the thermal treatment of the sample with zero external field. Charges originate due to thermionic emission from the electrodes and are injected into the dielectric by Richardson-Schottky process (Khare et al 1994b). In hygroscopic substances, which contain adsorbed and absorbed water 
molecules, SCE is mainly an activation controlled phenomenon. Although the exact nature of the activation centres is not clear, the manner in which the water molecules are accommodated in the polymer structure controls the mechanism of the emission process. The shape and magnitude of the transitional peaks and their location on the temperature scale depend on the heating rate because the polymer change their properties gradually over a certain interval of temperature (Shareef et al 1983). The first heating run empties all the activation centres and destroys some of them. The current during second subsequent heating is reproducible but it is much different from that of first heating in magnitude. It is because the activation centres are in the bulk of the polymer, which are due to absorbed water, are removed permanently by first heating run. A virgin sample is almost saturated with water and magnitude of current is maximum during first heating. Whenever dry samples were tried, it was found that the magnitude remained the same within experimental errors. The surface activation centres reappear by adsorbing moisture from the surroundings when the polymer is cooled below glass transition temperature $\left(T_{\mathrm{g}}\right)$ and they again produce weak current. The appearance of a strong peak in second heating run in SCE may confirm the detrapping mechanism of charge carriers/ions (Khare and Srivastava 1992b).

Due to active groups in PVP, the different pairs of opposite charges/ions as $\mathrm{H}^{+}$and $\mathrm{e}^{-}$or $\mathrm{H}^{+}$and $\mathrm{OH}^{-}$may be possible. $\mathrm{PVP}$ is known to be a highly hygroscopic material (Tan and Challa 1976). The water molecules may get absorbed in it from the surrounding conditions. The absorbed water molecules may form a weak complex with side group of PVP and a rise of current in SCE measurements in higher temperature range may be attributed to dissociation of this weak complex alongwith the liberation of ions/carriers. Several conflicting values from $140-175^{\circ} \mathrm{C}$ of rubber glass transition temperature $\left(T_{\mathrm{g}}\right.$ ) of PVP have been reported (Griffin 1968; Sidel 1970; Bandrup and Immergut 1975). According to Tan and Challa (1976), the different values of $T_{\mathrm{g}}$ may be due to difference in absorbed water content in PVP structure.They suggested the correct value of $T_{\mathrm{g}}$ of PVP from the equation

$$
\frac{1}{T_{\mathrm{g}}}=\frac{W(\mathrm{PVP})}{T_{\mathrm{g}}(\mathrm{PVP})}+\frac{W\left(\mathrm{H}_{2} \mathrm{O}\right)}{T_{\mathrm{g}}\left(\mathrm{H}_{2} \mathrm{O}\right)},
$$

where $T_{\mathrm{g}}\left(\mathrm{H}_{2} \mathrm{O}\right)$ represents the glass transition of water and $W\left(\mathrm{H}_{2} \mathrm{O}\right)$ the weight fraction of it and $T_{\mathrm{g}}$ (PVP) and $W$ (PVP) have the usual meanings. The polymer structure which accommodates the water traces and controls the mechanism of SCE may have the maximum rate of emission process at $T_{\mathrm{g}}$. In SCE the heating process could alter the quasi equilibrium position of dissociation of water as ions and carriers $\left(\mathrm{H}^{+}\right.$and $\left.\mathrm{OH}^{-}\right)$, and thus could lead to the movement of charge carriers/ ions. Further, the presence of dissolved oxygen molecules and hydrogen ions $\left(\mathrm{H}^{+}\right)$ makes the interface similar to that between an electronic conductor and an ionic conductor. The surface resistivity of polymers which absorb water is considerably decreased with relative humidity (Khare and Srivastava 1993). Elay and Leslie (1964) found a relationship between conductivity and weight of water absorbed. Absorbed water molecules may act as a source of ions, as plasticizer or as local structure modifier and also as electron donors (Seanor 1982). In following manner there may be the possibility of a complex formation between dissociated $\mathrm{OH}^{-}$ion of water and active carbonyl group. 


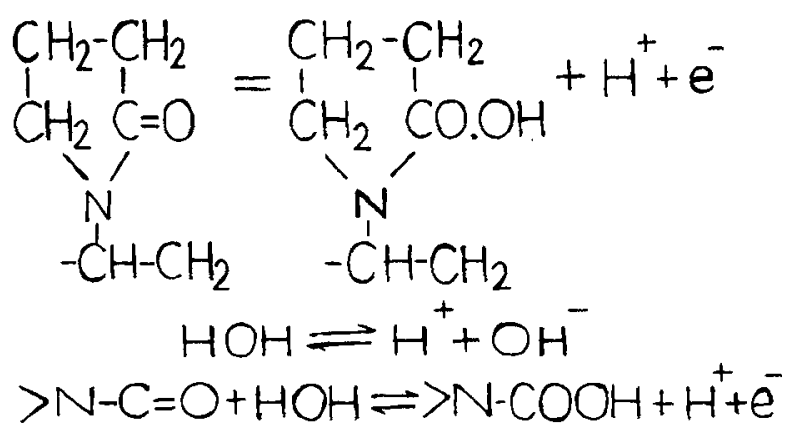

The complexes may get dissociated at higher temperature region, since polymer chains get loosened near $T_{\mathrm{g}}$, facilitating the increase of charge carriers resulting in a peak. Some of the charge carriers/ions may get trapped in different trapping levels of polymer. The PVP structure has distribution of traps due to carbonyl group of double bond and at $T_{\mathrm{g}}$ the mobility of segments of main chains will increase, facilitating the detrapping of bound charge carriers/ions. The justification of binding of water molecules in PVP in different phases of amorphous and crystalline regions, where absorption of water molecules occurs, can be understood by observing open circuit voltages $(\mathrm{OCV})$ at different electrode systems. The potential developed at an electrode seems to be a mixed one, comprising of (i) potential difference between the electrodes, (ii) charge exchange between a metal and dielectric due to difference in their work functions, and (iii) possible orientation of polar side group along with the water molecule as a dipole absorbed in PVP (Khare and Srivastava 1993). The hump of OCV vs temperature curves of first heating (figure 4) is, probably, because of the transitional changes in the structure of the polymer. During second heating OCV remains almost constant. In case of dissimilar electrode combinations, two electrode-dielectric interfaces have electrons exchange between them (charged layer), and for these combinations the work function differences of metals may have a controlling nature of $\mathrm{OCV}$ in magnitude. The primary source of charge carriers is the electrode contacts. Carriers are generated in the bulk on account of thermal ionization of impurities and absorbed water molecules. Nath and Kumar (1986) have shown injection of holes in cellulose acetate film and increase in charge mobility with iodine doping. Hole injection results in depletion of the layer of negative charges creating a field, at the interface between the electrode and the film surface. Considering a system consisting of dielectric sandwiches between electrodes is transferred from an initial state of pure capacitance to a final equilibrium state of pure resistance in series in the presence of charge carriers having different signs. The resistance of barrier layers and bulk material will be determined by the temperature condition of the system. Charges present in the barrier layers are driven off under the effect of the polarization field developed because of accumulation of charges in the barrier layers (Khare et al 1994). When two metals are brought in close contact, a potential difference of work function, $\phi_{2}-\phi_{1}$, is created between two metals. The semi-insulating polymer differs from a metal, however, in that an electric field may exist within the interior of the semi-insulator. For this reason the contact potential drop between the metal and the semi-insulator may take place within the material rather than at the contact interfaces. Along with the field, there may exist a depletion in the accumulation of charges in the surface layers. The work function of the polymeric 
material is higher compared to that of the metals used for contacts, which creates an accumulation layer at the interfaces and leaves charged donors in the bulk-faces of the metal-polymer system. The number of injected carriers may be larger for metals with lower work function such as aluminium and thus the field existing in the bulk may be partly compensated by the field created by injected charges at the interfaces. As the sample is heated, the charges existing at the interfaces have to pass through the bulk and may be trapped in the impurity centres and other trapping centres, such as chain coiling. The net charges injected from the electrodes are predominantly the majority centres. The charges which essentially give rise to the field at the interfaces are mobilized and the field due to injected charge carriers is weakened, resulting in the release of partly compensated bulk field and the motion of charge carriers is then determined by the field in the bulk (Khare and Srivastava 1992a).

\section{Conclusion}

Considering all these characteristics, we can conclude that the magnitude of current depends on the choice and combination of electrode metals, and observed spontaneous current is due to an electromotive force arising from an electrochemical process at the metal-polymer interface. The $\mathrm{M}_{1}-\mathrm{M}_{2}$ electrode systems which give current on heating may be termed as thermogalvanic cell.

\section{References}

Bandrup J and Immergut K (eds) 1975 Polymer hand book I-II (New York: Wiley) 2nd ed. p. 111 Chatterjee S K and Sethi K R 1983 J. Polym. Chem. 211050

Elay D D and Spirey D 1962 Trans. Faraday Soc. 58405

Elay D D and Leslie D 1964 Adv. Chem. Phys. 7238

Griffin in O Lewis 1968 Physical constants of linear homopolymers (Berlin: Springer-Verlag) p. 128

Gyoory A S 1965 in Horizon in biochemistry (eds) M Kasha and B P Pullenan (New York: Academic Press) p. 364

Khare P K and Srivastava A P 1992a Indian J. Pure \& Appl. Phys. 30131

Khare P K and Srivastava A P 1992b Thin Solid Films 208233

Khare P K and Srivastava A P 1993 Indian J. Pure \& Appl. Phys. 31399

Khare P K and Jain S K 1997 J. Polym. Mater. 1465

Khare P K, Keller J M and Datt S C 1992 Indian J. Pure \& Appl. Phys. 30768

Khare P K, Alka Bajpai and Srivastava A P 1993 Indian J. Pure \& Appl. Phys. 31405

Khare P K, Chandok R S, Dubey Neeraj and Srivastava A P 1994a Polym. Int. 35153

Khare P K, Vishwakarma H L and Srivastava A P 1994b Indian J. Phys. 68571

Nath R and Kumar A 1986 IEEE Trans. Electrical Insulators E-1 333

Seanor D A 1982 in Electrical conduction in polymers-electrical properties of polymers (New York: Academic Press)

Shareef A U, Saraf K K and Srivastava A P 1983 Phys. Status Solidi a77 381

Sidel Kovakaya F P 1970 The chemistry of N-vinyl pyrrolidone and its derivatives (Moscow: Nauka) 5

Tan Y Y and Challa G 1976 Polymer 17739 